\title{
Reviews and publications received
}

Sortiment wilder und kultivierter Kartoffelspecies - Teil 2

Untersuchungsergebnisse des Institutes für Pflanzenzüchtung Gross-Lüsewitz (GLKS) - D.D.R.

Bearbeitet von Dr. agr. D. Rothacker. 